\title{
JOINT CONTINUITY \\ OF SEPARATELY CONTINUOUS MAPPINGS WITH VALUES IN COMPLETELY REGULAR SPACES
}

Volodymyr Maslyuchenko - Oksana MyronyK — Olha Filipchuk

\begin{abstract}
We prove general theorems on quasi-continuity of mappings $f: X_{1} \times \cdots \times X_{n} \rightarrow Z$ with values in a completely regular space $Z$. As consequences, we obtain results on joint continuity of separately continuous functions of several variables involving the previous results of several authors.
\end{abstract}

\section{History}

The problem of joint continuity of separately continuous mappings has been intensively studied by mathematicians throughout the 20th century since the thesis of R. B a ir e [2] (see, surveys [37, [55], [56]). The research is currently ongoing (see dissertations [23, 26], [47]). Initially, results in this direction dealt with separately continuous mappings with values in metrizable spaces. There was one exception: Hoffmann-Jorgensen's example [8, p. 459] of everywhere discontinuous separately continuous mapping $f:[-1,1]^{2} \rightarrow[-1,1]^{[-1,1]^{2}}, f(x, y)(u, v)=$ $s p(u-x, v-y)$, where $s p(t, s)=2 t s /\left(t^{2}+s^{2}\right)$ for $(t, s) \neq(0,0)$ and $s p(0,0)=0$ is the famous Schwartz function. It takes values in the non-metrizable compact space $[-1,1]^{[-1,1]^{2}}$ endowed with the topology of pointwise convergence.

A lot of papers concerning joint continuity of separately continuous mappings and their analogues with values in generalized metric spaces have appeared during the last 25 years. First results were obtained for strict inductive limits 24, 25. (see also 33]), then for $\sigma$-metrizable and strong $\sigma$-metrizable spaces [10], 27], 31], 32], [34], [36], 37], further - for Moore spaces [35], [57, 58]. These results were included in dissertations [11, [26]. Recent papers [3], [20], 38], [40], [48], 49], [50], [51] concern stratifiable, semi-stratifiable and Maslyuchenko range spaces.

(C) 2017 Mathematical Institute, Slovak Academy of Sciences.

2010 Mathematics Subject Classification: 54C05, 54C08.

Keywords: separately continuous mapping, quasi-continuous mapping. 
New results in this branch were presented at the IV Conference devoted to the 135 anniversary of the birth of the famous Austrian mathematician Hans Hahn [12, 49]. They concerned separately continuous functions of several variables with values in the Ceder plane [49] and the Niemytski plane or, more generally, in completely regular Moore spaces [12].

Techniques used to obtain these results are similar and they require a common approach. The aim of this paper is to provide such an approach. The main results of it were announced in [41.

\section{The Weston property, the Hahn property, $W$ - and $H$-triples}

Let $X, Y$ and $Z$ be topological spaces and $f: X \times Y \rightarrow Z$ be a mapping. We denote by $C(f)$ the set of joint continuity points of $f$. Recall that a subset $E$ of a topological space $T$ is called residual in $T$ if its complement $T \backslash E$ is a set of the first category.

It is said that $f: X \times Y \rightarrow Z$ has the Weston property if for each $y \in Y$ the set

$$
C_{y}(f)=\{x \in X:(x, y) \in C(f)\}
$$

is residual in $X$. The mapping $f: X \times Y \rightarrow Z$ has the Hahn property if the set

$$
C_{Y}(f)=\{x \in X:\{x\} \times Y \subseteq C(f)\}
$$

is residual in $X$. This terminology was introduced in [26] because J. W e st o n [60] and $\mathrm{H}$. Hah n [16], [17] found that separately continuous mappings have the corresponding properties under certain assumptions.

Recall [21, 52] that a mapping $f: X \rightarrow Y$ is quasi-continuous at a point $x_{0}$ if for each neighborhood $V$ of $y_{0}=f\left(x_{0}\right)$ in $Y$ and each neighborhood $U$ of $x_{0}$ in $X$ there exists a non-empty open set $G$ in $X$ such that $G \subseteq U$ and $f(G) \subseteq V$. We say that $f$ is quasi-continuous if it is quasi-continuous at every point of $X$. The quasi-continuity of separately continuous functions of two real variables was discovered by V. Volte r r a [2, p. 94]; H. H a hn [15] was the first who applied it to study separately continuous functions of $n$ variables, however, the term "quasi-continuity" is due by S. K e m p ist y [21].

Let $f: X \times Y \rightarrow Z$ be a mapping and $p=(x, y) \in X \times Y$. Denote

$$
f^{x}(y)=f_{y}(x)=f(p) .
$$

The mappings $f^{x}: Y \rightarrow Z$ and $f_{y}: X \rightarrow Z$ are called $x$-section and $y$-section of the mapping $f$, respectively. A mapping $f: X \times Y \rightarrow Z$ is said to be a $K C$ -function, if for each $y \in Y$ the mapping $f_{y}: X \rightarrow Z$ is quasi-continuous and for each $x \in X$ the mapping $f^{x}: Y \rightarrow Z$ is continuous. $K C$-functions naturally 
arise while studying separately continuous functions $f: X_{1} \times \cdots \times X_{n+1} \rightarrow Z$ of several variables. One can interpret them as mappings $f: X \times Y \rightarrow Z$, where $X=X_{1} \times \cdots \times X_{n}$ and $Y=X_{n+1}$. Here, the mappings $f: X \times Y \rightarrow Z$ are often $K C$-functions, because separately continuous mappings are often quasi-continuous. So, an important problem is to describe the size of the set $C(f)$ for $K C$-functions $f: X \times Y \rightarrow Z$. This problem was studied in many papers (see dissertations [11], 23], 26], 47], [53], surveys [37, [55], [56] and references therein, and also recent results [5], 43], 44] that generalize those of [6], [42]).

Now, we introduce the main definitions. A triple $(X, Y, Z)$ of topological spaces is called a $W$-triple (H-triple) if each $K C$-function $f: X \times Y \rightarrow Z$ has the Weston property (the Hahn property). A space $Y$ is called a $W$-space (H-space) with respect to a space $Z$ if for any Baire space $X$ the triple $(X, Y, Z)$ is a $W$-triple ( $H$-triple). A $W$-space $(H$-space) with respect to the real line $\mathbb{R}$ is called a $W$-space (H-space). $H$-spaces were introduced in [26] (as Kempisty spaces) and studied in [47.

Theorem 2.1. A space $Y$ is a $W$-space (H-space) with respect to $Z$ if and only if for any topological space $X$ the triple $(X, Y, Z)$ is a $W$-triple (H-triple).

P r o of. Let $Y$ be a $W$-space ( $H$-space) with respect to $Z$ and $X$ be any topological space. The Banach category theorem [22, p. 87] implies that any topological space $X$ possesses the Baire kernel, i.e., the unique open Baire subspace $T$ with meager complement in $X$. Let $f: X \times Y \rightarrow Z$ be any $K C$-function. Then, its restriction $g=\left.f\right|_{T \times Y}: T \times Y \rightarrow Z$ is also a $K C$-function because the set $T$ is open in $X$. It follows from the definition that the function $g$ has the Weston property (Hahn property). Since $T$ is open, it is easily deduced that $C_{y}(g)=C_{y}(f) \cap T$ for each $y \in Y$ and $C_{Y}(g)=C_{Y}(f) \cap T$. But, if the trace $A \cap E$ of a set $A \subseteq X$ on a residual in $X$ set $E$ is residual in the subspace $E$, then the set $A$ is residual in $X$. Therefore, $f$ has the Weston property (Hahn property), and hence, $(X, Y, Z)$ is a $W$-triple ( $H$-triple).

\section{Examples of $W$-triples}

We formulate a general result which provides many examples of $W$-triples. To understand it better, recall some definitions.

A topological space $Z$ is called strongly $\sigma$-metrizable if $Z$ can be written as the union of an increasing sequence $\left(Z_{n}\right)_{n=1}^{\infty}$ of closed metrizable subspaces such that each convergent sequence in $Z$ is contained in some $Z_{n}$. The concept of a strongly $\sigma$-metrizable space was introduced in the survey [37.

A sequence $\mathcal{W}_{n}$ of open covers of a space $Z$ is a development for $Z$ if for each $z \in Z$ and each sequence of open sets $W_{n}$, such that $z \in W_{n} \in \mathcal{W}_{n}$ for all $n$, 
the family $\left\{W_{n}: n \in \mathbb{N}\right\}$ is a base at $z$. A regular space which has a development is called a Moore space. The Niemytski plane [9, p. 47] is an example of a nonmetrizable Moore space. We denote the Niemytski plane by $\mathbb{P}$.

A system $\mathcal{P}$ of open subsets of $X$ is called a pseudobase for $X$, if for every nonempty open set $U$ in $X$ there is a nonempty set $P \in \mathcal{P}$ with $P \subseteq U$.

In [7], J. C e d e r gave an example of a stratifiable and non-metrizable space [7. Ex. 9.1]. We call it the Ceder plane and denote by $\mathbb{M}$. A generalization of this construction known as the Ceder product was introduced in 39.

Theorem 3.1. A triple $(X, Y, Z)$ of topological spaces is a $W$-triple if the space $Y$ is first-countable, and at least one of the following conditions holds:

(i) $Z$ is metrizable;

(ii) $Z$ is strongly $\sigma$-metrizable;

(iii) $Z$ is a Moore space;

(iv) $X$ has a countable pseudobase, $Y$ is a connected Baire space, and $Z=\mathbb{M}$ is the Ceder plane.

P r o of. The sufficiency of (i) is a well-known result that can be obtained even using the methods of $\mathrm{H}$. $\mathrm{Hahn} \mathrm{[15],} \mathrm{its} \mathrm{proof} \mathrm{is} \mathrm{given,} \mathrm{for} \mathrm{example,} \mathrm{in} \mathrm{[28].}$ The sufficiency of condition (ii) was proved in [27]. The sufficiency of condition (iii) was shown in [57, and a more general result was obtained in [35. As for condition (iv), the corresponding result was proved in [40].

In particular, Theorem 3.1 (i) yields that any first-countable space is a $W$-space.

\section{Examples of $H$-triples}

We summarize the previously obtained results in the following statement. In this theorem the term "a Kempisty space" means "an $H$-space".

Theorem 4.1. A triple $(X, Y, Z)$ is an H-triple, if at least one of the following conditions holds:

(i) $Y$ is second-countable and $Z$ is metrizable;

(ii) $Y$ is a metrizable compact and $Z$ is strongly $\sigma$-metrizable;

(iii) $Y$ is a Kempisty compact and $Z$ is metrizable;

(iv) $Y$ is a Kempisty compact and $Z$ is strongly $\sigma$-metrizable;

(v) $X$ is a topological space which has a countable pseudobase, $Y$ is a connected Baire space, which satisfies the second axiom of countability, and $Z=\mathbb{M}$ is the Ceder plane. 
P r o o f. The sufficiency of condition (i) was proved in [28] and (ii) in [27]. The sufficiency of condition (iii) was obtained in [46, and (iv) in [10]. The sufficiency of condition (v) was proved in [40].

According to Theorem 4.1 (i), any second-countable space $Y$ is an $H$-space. $H$-triples can be used to define the notion of weakly Namioka space [59]. Namely, a topological space $X$ is weakly Namioka if for any second-countable space $Y$ the triple $(X, Y, \mathbb{R})$ is an $H$-triple.

The concept of the $H$-triple was used in [3], 4]. Namely, $K C$-Maslyuchenko spaces were introduced by T. B a n a k h in [3] as topological spaces $Z$ such that for every second-countable space $Y$ and every topological space $X$ the triple $(X, Y, Z)$ is an $H$-triple. The class of $K C$-Maslyuchenko spaces is closed under many countable operations, in particular, countable Tychonoff products and strong countable unions, which implies that strongly $\sigma$-metrizable spaces are $K C$-Maslyuchenko.

In [4, T. B a n a kh defined and studied Piotrowski spaces. A topological space $Z$ is Piotrowski if for any topological space $X$ and the singleton $1=\{0\}$ the triple $(X, 1, Z)$ is an $H$-triple.

\section{Quasi-continuity of $K C$-functions}

We shall use the following result on the quasi-continuity of a function $f: X \rightarrow Y$ with values in a completely regular space $Y$, which has been proven in [30] even for a more general case.

LEMMA 5.1. Let $X$ be a topological space, $Y$ a completely regular space, and let $x_{0} \in X$. A function $f: X \rightarrow Y$ is quasi-continuous at $x_{0}$ if and only if, for every continuous function $g: Y \rightarrow \mathbb{R}$, the composition $h=g \circ f: X \rightarrow \mathbb{R}$ is quasi-continuous at $x_{0}$.

We need the following simple assertion.

Lemma 5.2. Let $X$ be a Baire space, $Y$ be a $W$-space and $f: X \times Y \rightarrow \mathbb{R}$ be a KC-function. Then, $f$ is quasi-continuous.

Proof. Consider an arbitrary point $p_{0}=\left(x_{0}, y_{0}\right) \in X \times Y$ and prove that $f$ is quasi-continuous at the point $p_{0}$. Fix an arbitrary $\varepsilon>0$ and arbitrary neighborhoods $U$ and $V$ of $x_{0}$ and $y_{0}$ in the spaces $X$ and $Y$, respectively. By the quasi-continuity of $f_{y_{0}}: X \rightarrow \mathbb{R}$ at the point $x_{0}$, there is a non-empty open set $G$ in $X$ such that $G \subseteq U$ and $\left|f_{y_{0}}(x)-f_{y_{0}}\left(x_{0}\right)\right|<\varepsilon / 2$ for each $x \in G$. Since $Y$ is a $W$-space, the function $f$ has the Weston property, which implies that the set $C_{y_{0}}(f)$ is residual in $X$. But $X$ is Baire, then every residual subset 
of $X$ is everywhere dense [29, p. 64], so $C_{y_{0}}(f) \cap G \neq \emptyset$, and hence, there is a point $x_{1} \in C_{y_{0}}(f) \cap G$. Since $x_{1} \in C_{y_{0}}(f), f$ is continuous at the point $p_{1}=\left(x_{1}, y_{0}\right)$. Moreover, $x_{1} \in G$ and the set $G$ is open in $X$. Then, there exist open neighborhoods $U_{1}$ and $V_{1}$ of the points $x_{1}$ and $y_{0}$ in the spaces $X$ and $Y$, respectively, with $U_{1} \subseteq G, V_{1} \subseteq V$ and

$$
\left|f(x, y)-f\left(x_{1}, y_{0}\right)\right|<\varepsilon / 2
$$

if $(x, y) \in W=U_{1} \times V_{1}$. The set $W$ is non-empty open in the product $X \times Y$ and $W \subseteq U \times V$, while for each point $p=(x, y) \in W$ the following inequalities hold

$$
\left|f(p)-f\left(p_{0}\right)\right| \leq\left|f(p)-f\left(p_{1}\right)\right|+\left|f\left(p_{1}\right)-f\left(p_{0}\right)\right|<\varepsilon / 2+\varepsilon / 2=\varepsilon .
$$

Indeed, if $x_{1} \in G$ then $\left|f\left(p_{1}\right)-f\left(p_{0}\right)\right|=\left|f_{y_{0}}\left(x_{1}\right)-f_{y_{0}}\left(x_{0}\right)\right|<\varepsilon / 2$. This means that the function $f$ is quasi-continuous at the point $p_{0}$.

The following result can be easily deduced from Lemmas 5.1 and 5.2 .

TheOREM 5.1. Let $X$ be a Baire space, $Y$ a $W$-space, $Z$ a completely regular space, and let $f: X \times Y \rightarrow Z$ be a KC-function. Then, $f$ is quasi-continuous.

P r o of. Let $g: Z \rightarrow \mathbb{R}$ be a continuous function and $h=g \circ f$. It is easy to check that $h: X \times Y \rightarrow \mathbb{R}$ is a $K C$-function, because the composition $\varphi \circ \psi$ of two continuous functions is continuous, and the composition of a continuous function $\varphi$ and a quasi-continuous function $\psi$ is quasi-continuous. The $K C$-function $h$ is quasi-continuous by Lemma 5.2, because $X$ is Baire and $Y$ is a $W$-space. Therefore, by Lemma 5.1, the function $f$ is quasi-continuous at any point $p_{0} \in X \times Y$, i.e., quasi-continuous.

\section{Quasi-continuity of separately continuous functions of several variables}

Denote by $\mathcal{B}$ the class of all Baire spaces and consider a kind of conjugate class $\mathcal{B}^{*}$ which consists of all topological spaces $Y$ such that the product $X \times Y$ is a Baire space for every $X \in \mathcal{B}$. It is known that the class $\mathcal{B}^{*}$ contains the following spaces: Baire spaces which have a countable pseudobase [18, c. 56] (see also [14, [54]), pseudo-complete or countably complete spaces [1], 14, $\alpha$-favorable spaces in the Choquet game [61, and hereditarily Baire metric spaces [45] (see [19]). Note that $\mathcal{B}^{*} \subseteq \mathcal{B}$ but $\mathcal{B} \nsubseteq \mathcal{B}^{*}$, see [13], [54].

TheOREM 6.1. Let $X_{1}, X_{2}, \ldots, X_{n+1}$ be topological spaces for which $X_{1} \in \mathcal{B}$, let $X_{2}, \ldots, X_{n} \in \mathcal{B}^{*}, X_{2}, \ldots, X_{n+1}$ be $W$-spaces, $Z$ a completely regular space, and let $f: X_{1} \times X_{2} \times \cdots \times X_{n+1} \rightarrow Z$ be a separately continuous function. Then, $f$ is quasi-continuous. 
P r o of. We use induction on $n$. For $n=1$, we have two spaces $X_{1}$ and $X_{2}$ and a separately continuous mapping $f: X_{1} \times X_{2} \rightarrow Z$, where $X_{1}$ is a Baire space, $X_{2}$ is a $W$-space and $Z$ is a completely regular space. Since any continuous mapping is quasi-continuous, $f$ is a $K C$-function. Therefore, $f$ is quasi-continuous by Theorem 5.1 .

For the induction step, let $n \geq 2$ and suppose that the statement of the theorem is true for the number of spaces equal to $n$ and prove the assertion for $n+1$ spaces. Let $X=X_{1} \times \cdots \times X_{n}$ and $Y=X_{n+1}$. Since $X_{2}, \ldots, X_{n} \in \mathcal{B}^{*}$ and $X_{1} \in \mathcal{B}$, we consequently obtain $X_{1} \times X_{2} \in \mathcal{B}, X_{1} \times X_{2} \times X_{3} \in \mathcal{B}, \ldots$ $\ldots, X_{1} \times \cdots \times X_{n} \in \mathcal{B}$, therefore, $X \in \mathcal{B}$. By the assumption, $Y$ is a $W$-space. We shall consider $f$ as a function of two variables $x=\left(x_{1}, \ldots, x_{n}\right) \in X$ and $y=x_{n+1} \in Y$, while

$$
f(x, y)=f\left(x_{1}, \ldots, x_{n}, x_{n+1}\right) .
$$

For any fixed $y \in Y$, the function $f_{y}: X \rightarrow Z$ defined by $f_{y}(x)=f\left(x_{1}, \ldots, x_{n}, y\right)$, where $x=\left(x_{1}, \ldots, x_{n}\right) \in X$, is separately continuous as well as the function $f$. Therefore, by the induction assumption, the function $f_{y}$ is quasi-continuous. Since the function $f^{x}(y)=f(x, y)$ is continuous for each $x \in X, f$ is a $K C$ -function. By Theorem [5.1, the function $f$ is quasi-continuous on the product $X \times Y$, and hence on the product $X_{1} \times \cdots \times X_{n} \times X_{n+1}$.

\section{Main results}

Now, we are ready to get the main result.

Theorem 7.1. Let $X_{1} \in \mathcal{B}, X_{2}, \ldots, X_{n} \in \mathcal{B}^{*}$, let $X_{2}, \ldots, X_{n+1}$ be $W$-spaces, let $Z$ be a completely regular space, let $f: X_{1} \times X_{2} \times \cdots \times X_{n+1} \rightarrow Z$ be a separately continuous function, $X=X_{1} \times X_{2} \times \cdots \times X_{n}$ and $Y=X_{n+1}$. Then

(i) if $X_{n+1}$ is a $W$-space with respect to $Z$, then $(X, Y, Z)$ is a $W$-triple and $f: X \times Y \rightarrow Z$ has the Weston property;

(ii) if $X_{n+1}$ is an $H$-space with respect to $Z$, then $(X, Y, Z)$ is an $H$-triple and $f: X \times Y \rightarrow Z$ has the Hahn property.

P r o of. We again consider the function $f: X_{1} \times \cdots \times X_{n+1} \rightarrow Z$ as a function of two variables $x=\left(x_{1}, \ldots, x_{n}\right) \in X$ and $y=x_{n+1} \in Y$ putting

$$
f(x, y)=f\left(x_{1}, \ldots, x_{n}, x_{n+1}\right) .
$$

For every fixed $y \in Y$, the function $f_{y}: X \rightarrow Z$ is separately continuous, and therefore, is quasi-continuous by Theorem 6.1. Since all functions $f^{x}: Y \rightarrow Z$ are continuous for every $x \in X, f: X \times Y \rightarrow Z$ is a $K C$-function. The product $X$ is a Baire space, because $X_{1} \in \mathcal{B}$ and $X_{2}, \ldots, X_{n} \in \mathcal{B}^{*}$. Suppose $Y=X_{n+1}$ is a $W$-space with respect to $Z$. Then, $(X, Y, Z)$ is a $W$-triple by definition and 
$f: X \times Y \rightarrow Z$ is a $K C$-function with the Weston property. If $Y$ is an $H$-space with respect to $Z$ then $(X, Y, Z)$ is an $H$-triple and $f$ has the Hahn property.

One can obtain a stronger result for first-countable spaces using Banach category theorem.

Theorem 7.2. Let $X_{1}, X_{2}, \ldots, X_{n+1}$ and $Z$ be topological spaces, $X_{2}, \ldots, X_{n}$ be first-countable spaces, $X_{n+1}$ be a $W$-space, $Z$ be a completely regular space, $f: X_{1} \times X_{2} \times \cdots \times X_{n+1} \rightarrow Z$ be a separately continuous function, $X=X_{1} \times$ $X_{2} \times \cdots \times X_{n}$ and $Y=X_{n+1}$. Then:

(i) if $Y$ is a $W$-space with respect to $Z$ then $f: X \times Y \rightarrow Z$ has the Weston property;

(ii) if the product $X$ is a Baire space then $f$ is a quasi-continuous mapping;

(iii) if $Y$ is an $H$-space with respect to $Z$ then $f: X \times Y \rightarrow Z$ has the Hahn property.

P r o of. Properties (i) and (ii) will be proved by induction on $n$ simultaneously. If $n=1$ then we have $X=X_{1}$ and $Y=X_{2}$. Property (i) follows directly from the definition of a $W$-space with respect to $Z$ and Theorem 2.1. Property (ii) follows from Theorem 5.1 , because every separately continuous function is also a $K C$-function.

For the induction step, let $n \geq 2$ and suppose, that properties (i) and (ii) hold if the number of spaces is equal to $n$. Prove (i) and (ii) in the case where the number of spaces is equal to $n+1$, as in the Theorem statement. Let $T$ be the Baire kernel of $X$. The function $f$ is considered as a function of two variables $x=\left(x_{1}, \ldots, x_{n}\right) \in X$ and $y \in Y=X_{n+1}$ putting

$$
f(x, y)=f\left(x_{1}, \ldots, x_{n}, x_{n+1}\right) .
$$

Let $g=\left.f\right|_{T \times Y}$ be a restriction of $f$ to the product $T \times Y$. We claim that $g: T \times Y \rightarrow Z$ is a $K C$-function. Since $f$ is continuous with respect to the last variable $x_{n+1}$, the $x$-section $g^{x}: Y \rightarrow Z$ is continuous. Fix $y \in Y$ and prove that the $y$-section $g_{y}: T \rightarrow Z$ is quasi-continuous. Let $x_{0}=\left(x_{1}^{0}, \ldots, x_{n}^{0}\right) \in T$. Since the set $T$ is open in $X$, for every $k=1, \ldots, n$ there exists an open neighborhood $U_{k}$ of $x_{k}^{0}$ in $X_{k}$ such that $U=U_{1} \times \cdots \times U_{n} \subseteq T$. The product $U$ is Baire, because the kernel $T$ is a Baire space, and $U$ is its open subspace. Consider the restriction $h=\left.g\right|_{U \times Y}$ and its $y$-section $h_{y}: U \rightarrow Z$. The subspaces $U_{2}, \ldots, U_{n}$ are first-countable, because $X_{2}, \ldots, X_{n}$ are first-countable. Moreover, $U_{2}, \ldots, U_{n}$ are $W$-spaces, and so is $U_{n}$. Since $U$ is Baire and $h_{y}: U \rightarrow Z$ is separately continuous, $h_{y}$ is quasi-continuous by the induction assumption and (ii). In particular, $h_{y}$ is quasi-continuous at the point $x_{0}$. Since the set $U$ is open in $T$ and $\left.g_{y}\right|_{U}=h_{y}$, the function $g_{y}$ is quasi-continuous at the point $x_{0}$. Since $x_{0}$ is an arbitrary point of $T, g_{y}$ is quasi-continuous, and hence $g$ is a $K C$-function. The space $T$ is Baire 
and $Y$ is a $W$-space with respect to $Z$. So, the triple $(T, Y, Z)$ is a $W$-triple and the function $g$ has the Weston property. Since the set $T$ is residual in $X$ and $C_{y}(f) \supseteq C_{y}(g)$, for every $y \in Y$ (because $T$ is open in $X$ ), all the sets $C_{y}(f)$ are residual in $X$, thus, $f$ has the Weston property. If $X$ is a Baire space, then $T=X$ and $f=g$ is a $K C$-function, which is quasi-continuous by Theorem 2.1.

Prove property (iii) by induction on $n$. If $n=1$, this property follows from the definition of an $H$-space with respect to $Z$ and Theorem 2.1

Let $n \geq 2$. Suppose that (iii) is true for the number of spaces is equal to $n$, and we shall prove it for the number of spaces is equal to $n+1$. Consider the Baire kernel $T$ of the product $X$ and the restriction $g=\left.f\right|_{T \times Y}$. For every $y \in Y$, the mapping $g_{y}: T \rightarrow Z$ is quasi-continuous at any point $x_{0}=\left(x_{1}^{0}, \ldots, x_{n}^{0}\right)$ of $T$. Indeed, we can find open neighborhoods $U_{k}$ of $x_{k}^{0}$ in $X_{k}$ such that $U=$ $U_{1} \times \cdots \times U_{n} \subseteq T$. The restriction $\left.g_{y}\right|_{U}$ is separately continuous and the product $U$ is a Baire space, so $U_{1} \times \cdots \times U_{n-1}$ is a Baire space. Then, the restriction $\left.g_{y}\right|_{U}$ is quasi-continuous by (ii), which implies that $g_{y}$ is quasi-continuous at $x_{0}$. Moreover, $g^{x}: Y \rightarrow Z$ is obviously continuous, hence $g: T \times Y \rightarrow Z$ is a $K C$ function. The function $g$ has the Hahn property, since $(T, Y, Z)$ is an $H$-triple. Observe that $C_{Y}(f) \supseteq C_{Y}(g)$, because the set $T \times Y$ is open in the product $X \times Y$. Then,

$$
X \backslash C_{Y}(f) \subseteq X \backslash C_{Y}(g)=(X \backslash T) \cup\left(T \backslash C_{Y}(g)\right) .
$$

The set $X \backslash T$ is of the first category in $X$, and $T \backslash C_{Y}(g)$ is of the first category in $T$, and hence in $X$. Then, the complement $X \backslash C_{Y}(f)$ is of the first category in $X$, and $C_{Y}(f)$ is a residual set in $X$.

Note that the results of papers [49] and [12] can be obtained from Theorem 7.1 , but the Hahn property in Theorem 3.1 of $[12$ has to be replaced with the Weston property. Some of the results of [27] and [10] for separately continuous functions follow from Theorem 7.2 .

Acknowledgements. The authors are sincerely grateful to referee for his valuable comments that improved the manuscript.

\section{REFERENCES}

[1] AARTS, J. M.-LUTZER, D. J.: Pseudo-completeness and the product of Baire spaces, Pacific J. Math. 48 (1973), 1-10.

[2] BAIRE, R.: Sur les fonctions de variables réelles, Ann. Mat. Pura Appl. (4) 3 (1899), 1-123.

[3] BANAKH, T.: Quasicontinuous and separately continuous functions with values in Maslyuchenko spaces, Topology Appl. (to appear); preprint (http://arxiv.org/abs/1506.01661). 


\section{VOLODYMYR MASLYUCHENKO — OKSANA MYRONYK — OLHA FILIPCHUK}

[4] BANAKH, T.: Quasicontinuous functions with values in Piotrowski spaces, preprint (http://arxiv.org/abs/1609.05482).

[5] BOUZIAD, A.-TROALLIC, J. P.: Lower quasicontinuity, joint continuity and related concepts, Topology Appl. 157 (2010), 2889-2894.

[6] CAlBriX, J.-TROAlliC, J. P.: Applications séparément continues, C.R. Acad. Sc. Paris. Séc. A. 288 (1979), 647-648.

[7] CEDER, J.: Some generalizations of metric spaces, Pacif. J. Math. 11 (1961), 105-126.

[8] CHRISTENSEN, J. P. R.: Joint continuity of separately continuous functions, Proc. Amer. Math. Soc. 82 (1981), 455-461.

[9] ENGELKING, R.: General Topology. Mir, Moscow, 1986. (In Russian)

[10] FILIPCHUK, O. I.: On joint continuity of separately continuous mappings and its analogues with values in strongly $\sigma$-metrizable spaces, Nauk. Visn. Chernivets'kogo Univ., Mat. 336-337 (2007), 183-188. (In Ukrainian)

[11] FILIPCHUK, O. I.: Separately Continuous Mappings and Their Analogues with Values in Non-Metrizable Spaces. PhD Thesis, 2010. (In Ukrainian)

[12] FILIPCHUK, O. I.: Separately continuous functions with values in the Niemytski plane, in: Book of Abstracts, Int. Conf. dedicated to the 135th anniversary of Hans Hahn, Chernivtsi, Ukraine, 2014, pp. 205-206. (In Ukrainian)

[13] FleissneR, W. G.-KUNEN, K.: Barely Baire spaces, Fund. Math. 101 (1978), 229-240.

[14] FROLÍK, Z.: Baire space and some generalizations of complete metric spaces, Czechoslovak Math. J. 11 (1961), 237-248.

[15] HAHN, H.: Über Funktionen mehrerer Veränderlichen, die nach jeder einzelnen Veränderlichen stetig sind, Math. Zeitschr. 4 (1919), 306-313.

[16] HAHN, H.: Theorie der Reellen Funktionen. 1. Band, J. Springer, Berlin, 1921.

[17] HAHN, H.: Reelle Funktionen. 1. Teil. Punktfunktionen. Akademische Verlagsgesellschaft M. B. H., Leipzig, 1932.

[18] HAWORTH, R. C.-MCCOY, R. A.: Baire spaces, Dissertationes Math. (Rozprawy Mat.) 141 (1977), 1-77.

[19] HERNÁNDEZ, C.-MEDINA, L. R.-TKACHENKO, M.: Baire property in product spaces, Appl. Gen. Topol. 16 (2015), 1-13.

[20] KARlova, O. O.-MASLYUChEnKO, V. K.-MYRONYK, O. D.: The Bing plane and separately continuous mappings, Mat. Stud. 38 (2012), 188-193. (In Ukrainian)

[21] KEMPISTY, S.: Sur les fonctions quasicontinues, Fund. Math. 19 (1932), 184-197.

[22] KURATOWSKI, K.: Topology, Tom 1. Mir, Moscow, 1966. (In Russian)

[23] MASlyuChenKO, O. V.: Construction of $\omega$-Primitives and Some Analogues of Compact Operators. Doctoral Thesis, 2012. (In Ukrainian)

[24] MASLYUCHENKO, V. K.: Separately continuous functions with values in strict inductive limits, in: XIV School on Theory of Operators in Functional Spaces, P. II, Novgorod, Russia, 1989. (In Russian)

[25] MASLYUCHENKO, V. K.: Separately continuous functions with values in inductive limits, Ukraïn. Mat. Zh. 44 (3) (1992), 380-384. (In Ukrainian)

[26] MASLYUCHENKO, V. K.: Separately Continuous Mappings and Köthe Spaces. Doctoral Thesis, 1999. (In Ukrainian)

[27] MASLYUCHENKO, V. K.: Separately continuous functions of many variables with values in $\sigma$-metrizable spaces, Nonlinear Oscil. 2 (1999), 337-344. (In Ukrainian)

[28] MASLYUCHENKO, V. K.: Hahn spaces and Dini's problem, J. Math. Sci. (New York) 107 (2001), 3577-3582.

[29] MASLYUCHEnKO, V. K.: First Types of Topological Vector Spaces. Ruta, Chernivtsi, 2002. (In Ukrainian) 


\section{SEPARATELY CONTINUOUS MAPS WITH VALUES IN COMPLETELY REGULAR SPACES}

[30] MASLYUCHENKO, V. K.: On separate and joint modifications of continuity, Mat. Stud. 25 (2006), 213-218. (In Ukrainian)

[31] MASLYUChenKO, V. K.-FILIPCHUK, O. I.: Pointwise discontinuity of $K_{h} K-$ -functions with values in $\sigma$-metrizable spaces, Nauk. Visn. Chernivets'kogo Univ., Mat. 191-192 (2004), 103-106. (In Ukrainian)

[32] MASLYUCHENKO, V. K.-FILIPCHUK, O. I.: On the point discontinuity of $K_{h} C$ -mappings on continuous curves, Nauk. Visn. Chernivets'kogo Univ., Mat. 314-315 (2006), 122-124. (In Ukrainian)

[33] MASLYUCHENKO, V. K.-FILIPCHUK, O. I.: Regular sequences of normed spaces and joint continuity of separately continuous mappings, Bukovinian Math. J. 3 (2015), 115-119. (In Ukrainian)

[34] MASLYUCHENKO, V. K.-MYKHAYLYUK, V. V.-FILIPCHUK, O. I.: Points of joint continuity of separately continuous mappings with values in the Niemytski plane, Mat. Stud. 26 (2006), 217-221. (In Ukrainian)

[35] MASLYUCHENKO, V. K.-MYKHAYLYUK, V. V.-FILIPCHUK, O. I.: Joint continuity of $K_{h} C$-functions with values in Moore spaces, Ukraïn. Mat. Zh. 60 (2008), 1539-1547. (In Ukrainian)

[36] MASLYUCHENKO, V. K.-MYKHAYLYUK, V. V.-SHYSHYNA, O. I.: Joint continuity of horizontal quasi-continuous mappings with values in $\sigma$-metrizable spaces, Mat. Metody i Fiz.-Mekh. Pólya 45 (2002), 42-46. (In Ukrainian)

[37] MASLYUCHENKO, V. K.-MYKHAYLYUK, V. V.-SOBCHUK, O. V.: On investigation of separately continuous functions, in: Proceedings of Intern. Math. Conf. dedicated to the anniv. of Hans Hahn, 1995, 192-246. (In Ukrainian)

[38] MASLYUCHENKO, V. K.-MYRONYK, O. D.: Separately continuous mappings and stratifiable spaces, Math. Visnyk NTSH 7 (2010), 111-121. (In Ukrainian)

[39] MASLYUCHENKO, V. K.-MYRONYK, O. D.: The Ceder product and stratifiable spaces, Bukovinian Math. J. 1 (2013), 107-112. (In Ukrainian)

[40] MASLYUCHENKO, V. K.-MYRONYK, O. D.: On continuity of KC-functions with values in the Ceder plane, Carpathian Math. Publ. 6 (2014), 329-336.

[41] MASLYUCHENKO, V. K.-MYRONYK, O. D.-FILIPCHUK, O. I.: Separately continuous mappings with values in completely regular spaces, in: Book of Abstracts, XI Summer School "Algebra, Topology and Analysis", 2014, pp. 50-51. (In Ukrainian)

[42] MASLYUCHENKO, V. K.-NESTERENKO, V. V.: Joint continuity and quasi-continuity of horizontal quasi-continuous mappings, Ukraïn. Mat. Zh. 52 (2000), 1711-1714. (In Ukrainian)

[43] MASlyuchenkO, V. K.-NeSterenKO, V. V.: Points of joint continuity and large oscillations, Ukraïn. Mat. Zh. 62 (2010), 791-800. (In Ukrainian)

[44] MASLYUCHENKO, V.-NESTERENKO, V.: A new generalization of Calbrix-Troallic's theorem, Topology Appl. 164 (2014), 162-169.

[45] MOORS, W. B.: The product of a Baire with a hereditarily Baire metric space is Baire, Proc. Amer. Math. Soc. 134 (2006), 2161-2163.

[46] MYKHAYLYUK, V. V.: The Namioka property of KC-functions and Kempisty spaces, Topology Appl. 153 (2006), 2455-2461.

[47] MYKHAYLYUK, V. V.: Coordinate Method and Theory of Separately Continuous Mappings. Doctoral Thesis, 2009. (In Ukrainian)

[48] MYRONYK, O. D.: On separately continuous mappings with values in the Ceder plane, Bukovinian Math. J. 1 (2013), 100-105. (In Ukrainian)

[49] MYRONYK, O. D.: Separately continuous functions with values in the Ceder plane, in: Book of Abstracts, Int. Conf. dedicated to the 135th anniversary of Hans Hahn, Chernivtsi, Ukraine, 2014, pp. 131-132. (In Ukrainian) 


\section{VOLODYMYR MASLYUCHENKO — OKSANA MYRONYK — OLHA FILIPCHUK}

[50] MYRONYK, O. D.: On the separately continuous functions with values in the Ceder plane, Bukovinian Math. J. 2 (2014), 173-176. (In Ukrainian)

[51] MYRONYK, O. D.: Stratifiable, Semistratifiable Spaces and Separately Continuous Mappings. PhD Thesis, 2015. (In Ukrainian)

[52] NEUBRUNN, T.: Quasi-continuity, Real Anal. Exch. 14 (1988-1989), 259-306.

[53] NeSTEREnKO, V. V.: Different Types of Quasi-Continuity and its Applications. PhD Thesis, 1999. (In Ukrainian)

[54] OXTOBY, J. C.: Cartesian products of Baire spaces, Fund. Math. 49 (1960/1961), $157-166$.

[55] PIOTROWSKI, Z.: Separate and joint continuity, Real Anal. Exch. 11 (1985), 293-322.

[56] PIOTROWSKI, Z.: Separate and joint continuity II, Real Anal. Exch. 15 (1989), 248-256.

[57] PIOTROWSKI, Z.: Mibu-type theorem, in: Classical Analysis, Proc. Inter. Symp., 1993, Radom, Poland, 1993, pp. 141-147.

[58] PIOTROWSKI, Z.: On the theorems of Y. Mibu and G. Debs on separately continuity, Inter. J. Math. and Math. Sci. 19 (1996), 495-500.

[59] PIOTROWSKI, Z.-WALLER, R.: Baire and weakly Namioka spaces, Topology Appl. 159 (2012), 3294-3299.

[60] WESTON, J. O.: Some theorem on cluster sets, J. London Math. Soc. 33 (1958), 435-441.

[61] WHITE, H. E., JR.: Topological spaces that are $\alpha$-favorable for a player with perfect information, Proc. Amer. Math. Soc. 50 (1975), 477-482.

Received February 3, 2016

Yuriy Fedkovych

Chernivtsi National University

Department of Mathematical Analysis

Kotsyubyns'ky Str. 2

58012 Chernivtsi

UKRAINE

E-mail: math.analysis.chnu@gmail.com

Yuriy Fedkovych

Chernivtsi National University

College CHNU

Kotsyubyns'ky Str. 2

58012 Chernivtsi

UKRAINE

E-mail: myronyk.oks@gmail.com

Yuriy Fedkovych

Chernivtsi National University

Department of Mathematical Problems

of Control and Cybernetics

Kotsyubyns'ky Str. 2

58012 Chernivtsi

UKRAINE

E-mail: o-sh@ukr.net 